\title{
Randomised controlled trial of thiopental for intubation in neonates
}

\author{
A Bhutada, R Sahni, S Rastogi, J-T Wung
}

\begin{abstract}
Aims-To determine the effects of premedication with thiopental on heart rate, blood pressure, and oxygen saturation during semi-elective nasotracheal intubation in neonates.

Methods-A randomised, placebo controlled, non-blinded study design was used to study 30 neonates (mean birthweight $3.27 \mathrm{~kg}$ ) requiring semi-elective nasotracheal intubation. The babies were randomly allocated to receive either $6 \mathrm{mg} / \mathrm{kg}$ of thiopental (study group) or an equivalent volume of physiological saline (control group) one minute before the start of the procedure. Six infants were intubated primarily and 24 were changed from orotracheal to a nasotracheal tube. The electrocardiogram, arterial pressure wave, and transcutaneous oxygen saturation were recorded continuously 10 minutes before, during, and 20 minutes after intubation. Minute by minute measurements of heart rate, heart rate variability, mean blood pressure (MBP) and transcutaneous oxygen saturation $\left(\mathrm{SpO}_{2}\right)$ were computed. The differences for all of these between the baseline measurements and those made during and after intubation were determined. Differences in the measurements made in the study and the control groups were compared using Student's $t$ test.
\end{abstract}

Results-During intubation, heart rate increased to a greater degree $(12.0$ vs -0.5 beats per minute, $p<0.03$ ) and $\mathrm{MBP}$ increased to a lesser degree (-2.9 vs 4.4 mm Hg; p < 0.002) in the infants who were premedicated with thiopental. After intubation only the changes in MBP differed significantly between the two groups $(-3.8$ vs $4.6 \mathrm{~mm} \mathrm{Hg}$; $p$ 0.001). There were no significant changes in the oxygen saturation between the two groups during or after intubation. The time taken for intubation was significantly shorter in the study group $(\mathrm{p}<0.04)$.

Conclusions-The heart rate and blood pressure of infants who are premedicated with thiopental are maintained nearer to baseline values than those of similar infants who receive no premedication. Whether this lessening of the acute drop in the heart rate and increase in blood pressure typically seen during intubation of unmedicated infants is associated with long term advantages to the infants remains to be determined.

(Arch Dis Child Fetal Neonatal Ed 2000;82:F34-F37)

Keywords: heart rate; blood pressure; oxygen saturation
Endotracheal intubation for the management of respiratory failure or in anticipation of a surgical procedure is commonly done semielectively in awake patients in neonatal intensive care units. Intubation of the trachea and the associated direct laryngoscopy are powerful noxious stimuli with several potential adverse effects. For example, hypertension, tachycardia, and bradycardia are frequently observed cardiovascular responses to laryngoscopy or intubation in adults, and bradycardia and decreased oxygen saturation often accompany intubation in neonates. ${ }^{12}$ Other responses to laryngoscopy include laryngospasm, bronchospasm, and increased intracranial pressure. ${ }^{3}$ The changes in heart rate, blood pressure, oxygen saturation, and intracranial pressure associated with intubation may be the expression of accompanying pain, probably produced by autonomic overactivity. ${ }^{24-7}$ Alternatively, these cardiovascular changes may reflect reflex vagal activity induced by unusually intense stimulation of the naso- and oropharynx. Whatever the origin, these abnormal variations in vascular pressures and flows are likely to lead to changes in tissue oxygenation, and may be associated with increased risk of spontaneous intracerebral haemorrhage. Stabilisation of oxygenation and perfusion during semi-elective intubation is a reasonable objective.

Various anaesthetic agents such as fentanyl, propofol, midazolam, etc, have been used extensively during semi-elective intubation in adults and children to minimise or eliminate most of the adverse effects associated with intubation. ${ }^{8}$ However, there have been no randomised, controlled trials on the use of these agents for neonatal intubation and there is very little available information on the efficacy, safety, and the associated adverse effects of premedicating agents during intubation of neonates. In fact, most neonatal intubations are done with the child awake, and when premedication is used, the doses are probably too small to prevent most of the above adverse effects. This study aimed to test the hypothesis that pre-medication with thiopental reduces or eliminates the variation in heart rate, heart rate variability, blood pressure and oxygen saturation $\left(\mathrm{SpO}_{2}\right)$, induced by intubation. We also examined the effect of duration of the procedure on the cardiovascular responses.

\section{Methods}

Thirty neonates, admitted to neonatal intensive care at this hospital between January and September 1997, were studied. All neonates weighed more than $2 \mathrm{~kg}$ at birth and required semi-elective intubation for the management of 
Table 1 Population characteristics*

\begin{tabular}{llll}
\hline Characteristics & Control group $(n=13)$ & Study group $(n=14)$ & $p$ Value \\
\hline Birthweight (kg) & $3.30(0.53)$ & $3.45(0.97)$ & $>0.05$ \\
Gestational age (weeks) & $39.2(1.4)$ & $39.6(1.2)$ & $>0.05$ \\
$\begin{array}{l}\text { Sex (male/female) } \\
\text { Apgar (5 minute) }\end{array}$ & $9 / 6$ & $11 / 4$ & $>0.05$ \\
$\begin{array}{l}\text { Indication for intubation } \\
\begin{array}{l}\text { Respiratory failure } \\
\text { Preoperative }\end{array}\end{array}$ & $9(8-10)$ & $7(5-9)$ & $>0.05$ \\
$\quad 6$ cardiac & 6 & 7 & \\
$\quad$ surgical & 8 & 6 & \\
\hline
\end{tabular}

All values are mean (SD) except 5 minute Apgar scores which are expressed as median (range).

${ }^{\star}$ Data from 3 infants (control $=2$, study $=1$ ) were excluded from analysis.

respiratory failure or before general anesthesia for elective surgery. The study was approved by the Institutional Review Board and informed parental consent was obtained before enrolment.

The enrolled neonates were randomly allocated to study (thiopental) and control (placebo) groups using randomisation cards. The patient characteristics are given in table 1 . There were no significant differences in the demographic variables observed between the study and the control groups. All intubations were nasotracheal and of 30 neonates, 24 were studied during a change from an orotracheal to a nasotracheal tube.

A randomised, placebo controlled, nonblinded study design was used. Enrolled neonates were randomly allocated to two groups. The study group received thiopental 6 $\mathrm{mg} / \mathrm{kg}(0.24 \mathrm{ml} / \mathrm{kg}$ of a $2.5 \%$ solution) while the control group received an equivalent volume of physiological saline. The electrocardiogram (ECG), arterial pressure waveform, and transcutaneous oxygen saturation were continuously monitored and recorded $10 \mathrm{~min}$ utes before, during, and 20 minutes after intubation in all neonates.

All enrolled neonates were receiving intensive care and had indwelling arterial catheters at the time of the study. Continuous data were acquired using analogue outputs from an $\mathrm{HP}$ monitor (Hewlett Packard Model 54, M1175A, Englewood, CO, USA) and a pulse oximeter (Nellcor N-200, St Louis, MO, USA) using a 16 bit analogue to digital converter. The EKG signal was processed on-line and stored as $R R$ intervals using an RR pre-processor with an accuracy of 1 millisecond. The arterial pressure waveform and transcutaneous oxygen saturation analogue signals were digitised using sampling rates of $50 \mathrm{~Hz}$ and $25 \mathrm{~Hz}$, respectively. Raw digital data were stored in the bedside microcomputer for subsequent off-line analysis.

Baseline data were acquired for at least 10 minutes. When the nurse and physician were ready, thiopental or placebo was administered by slow intravenous infusion over 15 seconds. Nasotracheal intubation was then performed, beginning one minute after completion of the infusion to coincide with the peak effect of the thiopental. Data collection was then continued for a minimum of 20 minutes after intubation had been completed.

All intubations were performed by one of the two senior neonatal fellows, both certified regional trainers for the Neonatal Resuscitation Program. The appropriate length for the nasotracheal tube insertion was predetermined and marked, so that the tip lay between the vocal cords and carina based on crown-heel length. ${ }^{9}$ For purposes of data analysis, nasotracheal intubation was considered to have started with the introduction of an endotracheal tube into a nostril. With the aid of an Oxyscope blade and oxygen flowing at 2 litres/min, the tube was advanced toward the vocal cords. The oropharynx was then suctioned under direct vision. The tip of the endotracheal tube was grasped by an alligator forceps and guided through the vocal cords. For the change-over from oral to nasotracheal tubes, the orotracheal tube was left in place until the tip of the nasotracheal tube was grasped with the alligator forceps, at which point the oral tube was removed and the nasotracheal tube was guided immediately through the vocal cords. The nasotracheal tube was fixed with a single piece of tape. The time for intubation (TFI) was measured from the introduction of the nasotracheal tube into the nostril until its fixation with the tape.

\section{DATA PROCESSING}

Heart rate

The electrocardiogram was processed using a specially designed RR interval preprocessor. The preprocessor detects $\mathrm{R}$ waves, measures $R R$ interval ( $\pm 1 \mathrm{msec}$ ), and passes these times to the computer over a parallel port. Mean heart rate was computed as the inverse of the mean of RR intervals ( $\times 60000$ ) measured during each block of time. The standard deviation of all RR intervals, a global measure of heart rate variability, was also computed for each minute, using dedicated software.

\section{Blood pressure}

Using dedicated software, systolic and diastolic pressures were computed from the individual peaks and troughs of the arterial pressure waveform and were averaged to yield minute averages. Similarly, mean blood pressure was computed as the overall mean of the pressure signal.

\section{Oxygen saturation}

The analogue signal from the Nellcor N-200 pulse oximeter was also processed and averaged using similar software to yield a measurement of arterial saturation for each minute of the study.

The study was divided into four stages: stage I pre-intubation stage (baseline); stage II drug administration stage; stage III laryngoscopy/ intubation stage; stage IV post-intubation stage.

Minute by minute measurements of heart rate, heart rate variability, $\mathrm{MBP}$ and $\mathrm{SpO}_{2}$ from each of the above four stages were averaged to yield mean values for the individual stages. The mean values of the above variables and the time for intubation were then compared between the study and control groups using Student's $t$ test. Data obtained from two infants in the control group and one infant in the study group were lost during acquisition, and these three infants were not included in the analysis. 


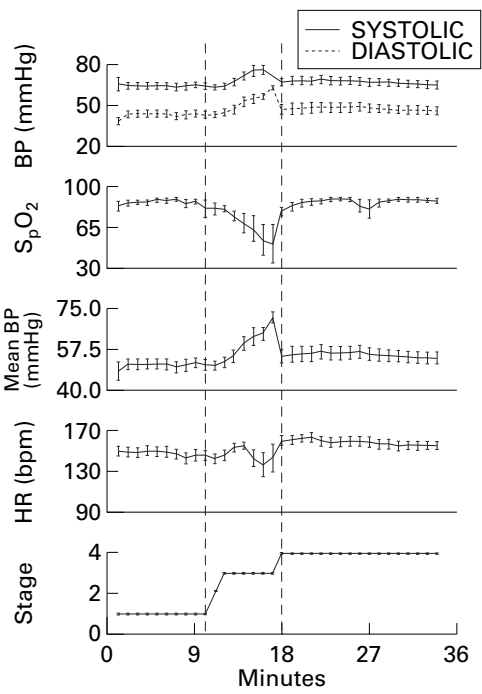

(A)

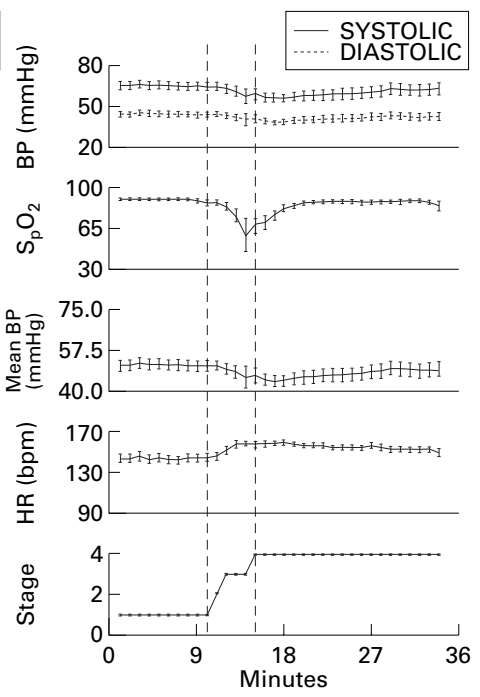

(B)
Figure 1 Ensemble of average minute to minute measurements of systolic and diastolic blood pressures, transcutaneous oxygen saturation $\left(\mathrm{SpO}_{2}\right)$, mean blood pressure and heart rate during four different stages of intubation for the control group $(A)$ and study group (B). Stage I pre-intubation baseline; stage II drug/placebo administration; stage III intubation period; and stage IV after intubation. Data are presented as mean (SE).

\section{Results}

Ensemble average, minute to minute measurements of heart rate, $\mathrm{MBP}$, and $\mathrm{SpO}_{2}$ are shown in fig 1 for both groups of neonates. Time trends are evident in most variables. Baseline measurements (stage I) and measurements during drug infusion (stage II) for all variables in both groups were comparable. During intubation (stage III), differences between the two groups were observed in heart rate and MBP. In the study group there was a gradual increase in heart rate and a slight fall in blood pressure while the control group showed a net decline in heart rate and a substantial increase in blood pressure. After intubation (stage IV) the difference between mean blood pressures of the medicated subjects and the controls persisted. The control subjects took longer to recover to baseline values.

Table 2 gives the statistical analysis of the changes in heart rate, heart rate variability, $\mathrm{MBP}$ and $\mathrm{SpO}_{2}$ from baseline to during intubation (stages I to III) and after intubation (stages I to IV) between the control and the study groups. During intubation significant changes from baseline were seen in heart rate (mean (SE) -0.5 (4.4) vs 12.0 (3.2) bpm; p < 0.03 ), heart rate variability (mean (SE) -2.0 (2) vs 19.0 (5) msec; p < 0.01 ), and MBP (mean (SE) 4.4 (1.1) vs -2.9 (1.8) $\mathrm{mm} \mathrm{Hg}$; $<0.002$ ) between the control and study groups, respectively. After intubation (stage IV) only the changes in MBP differed significantly between the control and study groups (mean (SE) 4.6 (1.2) vs -3.8 (1.8) $\mathrm{mm} \mathrm{Hg;} \mathrm{p} \mathrm{<}$ $0.001)$. There were no significant changes observed in the $\mathrm{SpO}_{2}$ between the two groups during or after intubation when compared with the baseline.

Time for intubation (TFI) was significantly longer (mean (SE) 5.08 (1.10) vs 2.70 (0.37); $\mathrm{p}<0.04)$ in the control group compared with the study group. Linear regression analysis of cardiovascular variables against TFI (stage III) showed significant positive correlations of TFI with heart rate variability $(\mathrm{r}=0.59 ; \mathrm{p}<0.04)$, and MBP ( $\mathrm{r}=0.71 ; \mathrm{p}<0.007)$ and a negative correlation between TFI and $\mathrm{SpO}_{2}(\mathrm{r}=0.78 ; \mathrm{p}$ $<0.002$ ) in the control group, while no significant relations were observed in the study group.

\section{Discussion}

Intubation with direct laryngoscopy, even in the most experienced hands and under ideal conditions, is associated with several potential stresses for newborn infants. Manipulation of the upper airway may result in temporary mechanical interruption of gas flow, activation of potentially maladaptive cardiovascular reflexes, activation of pain pathways, and voluntary and involuntary motor responses from the infant. As a result of one or more of these stresses, potentially unfavourable changes in heart rate, blood pressure, oxygen saturation and intracranial pressure have been observed. ${ }^{2-6}$ Adverse changes in these variables might, in turn, further compromise an already critically ill neonate. Most intubations in neonates are performed with the infant awake, without analgesia or muscle relaxation, possibly due to fear of producing cardiovascular or ventilatory depression. ${ }^{10}$ There is also a widespread belief that immature neonates experience less pain during the procedure and tolerate the procedure better than older patients.

It is difficult to measure pain in babies, but substantial evidence obtained from experimental animals indicates that pain during early life not only alters behaviour and physiology acutely ${ }^{11}$ but may also influence pain thresholds and responses to pain during subsequent development. ${ }^{12}$ Lack of myelination has been cited by some authors as evidence of immaturity of the neonatal nervous system, ${ }^{13-15}$ which, in turn might lead to decreased pain perception. Incomplete myelination merely implies a slower conduction velocity in the peripheral nerves and central nerve tracts of neonates, changes which may be offset completely by the shorter interneuron and neuromuscular dis-

Table 2 Changes in heart rate (HR), heart rate variability (HRV), mean blood pressure (MBP) and transcutaneous oxygen saturation $\left(\mathrm{SpO}_{2}\right)$ from baseline, during, and following intubation in study and control groups: data are presented as mean (SE)

\begin{tabular}{|c|c|c|c|c|c|c|}
\hline & \multicolumn{3}{|c|}{ During intubation } & \multicolumn{3}{|c|}{ After intubation } \\
\hline & Study & Control & $p$ & Study & Control & $p$ \\
\hline Changes in HR (bpm) & $12.0(3.2)$ & $-0.5(4.4)$ & $<0.03$ & $11.5(2.9)$ & $9.0(3.2)$ & 0.58 \\
\hline Changes in HRV (ms) & $-2.0(2.0)$ & $19.0(5.0)$ & $<0.01$ & $-1.8(1.2)$ & $-5.8(5.6)$ & 0.50 \\
\hline Changes in MBP (mm Hg) & $-2.9(1.8)$ & $4.4(1.1)$ & $<0.002$ & $-3.8(1.8)$ & $4.6(1.2)$ & $<0.001$ \\
\hline Changes in $\mathrm{SpO}_{2}(\%)$ & $-11.2(3.7)$ & $-14.6(5.3)$ & 0.18 & $-2.9(1.4)$ & $1.2(1.9)$ & 0.50 \\
\hline
\end{tabular}


tances travelled by the impulse. ${ }^{16}$ The changes in heart rate, blood pressure, oxygen saturation, and intracranial pressure associated with intubation are consistent with the hypothesis that the infant is experiencing pain. ${ }^{24-7}$ Whether endotracheal intubation is, in fact, painful, is not known, but the fact that fundamental physiological measurements seem to be favourably influenced by premedication suggests that judicious use of an analgesic/ anaesthetic agent during endotracheal intubation may be desirable.

Thiopental is one such agent and might exert its pharmacological effects by interfering with pain pathways. It probably acts through the gamma aminobutyric acid (GABA) receptor complex type A. ${ }^{17}$ It enhances and mimics the action of GABA, ${ }^{18}$ the principal inhibitory neurotransmitter of the mammalian central nervous system. Another possible explanation of the therapeutic effect of thiopental is that the drug abolishes the reflex vagal and sympathetic responses to manipulation of the oropharynx during intubation. Intubation activates vagal parasympathetic reflexes via stimulation of vagal afferent fibres in the pharynx, oesophagus, and the respiratory tract, ${ }^{19}$ which, in turn, may cause reflex sinus bradycardia. Increased sympathoadrenal activity has been hypothesised as the cause of hypertension during laryngoscopy and intubation. ${ }^{20}$ This may be attributable to mechanical reflexes aroused by pressure on the base of the tongue and epiglottis, probably via stimulation of the epipharyngeal and laryngopharyngeal region. Stimulation of this area is known to increase activity in the cervical sympathetic efferent fibres. ${ }^{21}$ In our study, the control group showed a mean decrease in heart rate from baseline during intubation (perhaps a direct effect on vagal reflexes) and an increase in blood pressure from baseline during the same period (perhaps mediated by the peripheral sympathetic nervous system). These alterations in autonomic activity are known to be ablated by the acute administration of thiopental. These changes were not seen in the study group who received thiopental before intubation.

Not surprisingly, given the size and maturity of the neonates in this study, pretreatment with thiopental was associated with a reduction in the time required for intubation. The correlation between the time required for intubation and the extent of the potentially unfavourable changes in the measurements argues for an additional advantage of thiopental administration.

The major clinical adverse effects of thiopental include a dose dependent depression of electrocortical activity ${ }^{22}$ and respiratory drive. Venous vasodilation has also been observed after thiopental administration, followed by pooling of peripheral blood..$^{23}$ In our study the tachycardia observed in the study population might be related to the slight fall in blood pressure. This drop in blood pressure was transient and did not require treatment. Blood pressure had returned to normal within 20 minutes. The fact that no other side effects were observed in the study group suggests that it could be safely used in this population before elective intubation.

In conclusion, the data obtained in this prospective, randomised controlled trial support the hypothesis that infants premedicated with thiopental maintain vital signs closer to baseline measurements than infants who were not premedicated. This study was done in a cohort of relatively large and mature neonates being treated for a variety cardiorespiratory and surgical diseases. Infants of different size and maturity and those with different diagnoses may behave differently. Whether the observed differences in cardiorespiratory stability are of significant long term advantage to the infant is not known. However, on the basis of the data obtained in this study, intubation following an induction dose of an anesthetic agent seems to be an easier and more humane strategy, and may spare the neonate needless distress.

1 Stone DJ, Gal TJ. Airway management. In: Miller RD, ed. Anesthesia. New York: Churchill Livingstone, 1994:1403-

2 Kelly MA, Finer NN. Nasotracheal intubation in neonates: physiological responses and effects of atropine and pancuronium. F Pediatr 1984;105:303-9.

3 Millar C, Bissonnette B. Awake intubation increases intracranial pressure without affecting cerebral blood flow velocity in infants. Can $\mathcal{F}$ Anaesth 1994;41:281-7.

4 Marshall TA, Deeder R, Pai S, Berkowitz GP, Austin TL. Physiologic changes associated with endotracheal intubation in preterm infants. Crit Care Med 1984;12:501-4.

5 Gibbons PA, Swedlow DB. Changes in oxygen saturation during elective tracheal intubation in infants. Anesth Analg 1987;66:874-8

6 Raju TNK, Vidyasagar D, Torres C, Grundy D, Bennett EJ. Intracranial pressure during intubation and anesthesia in infants. F Pediatr 1980;96:860-2.

7 Stevens BJ, Johnston CC, Grunau RVE. Issues of assessment of pain and discomfort. FOGNN 1995;24:849-55.

8 Barrington KJ, Byrne PJ. Premedication for neonatal intubation. Am f Perinatol 1998;15:213-21.

9 Coldiron JS. Estimation of nasotracheal tube length in neonates. Pediatrics 1968;41:823-7.

10 Richards T. Can a fetus feel pain? BMF 1985;291:1220-1.

11 Anand KJS. Relationships between stress responses and clinical outcome in newborn, infants and children. Crit Care Med 1993;21:S358-S9.

12 Menon G, Anand KJS, McIntosh N. Practical approach to analgesia and sedation in the neonatal intensive care unit. Semin Perinatol 1998; 22:417-24.

13 Tilney F, Rosett J. The value of brain lipoids as an index of brain development. Bull Neurol Inst NY 1931;1:28-71.

14 Shearer MH. Surgery on the paralyzed, unanesthetized newborn. Birth 1986;13:79.

15 Hatch DJ. Analgesia in the neonate. BMF 1987;294:920

16 Gestation, wachstum und hirnentwicklung. In: Linneweh F, eds. Fortschritte der Paedologie. Vol 2. Berlin: SpringerVerlag, 1968:46-64

17 Olson RW. Barbiturates. Int Anesthesiol Clin 1988;26:254

18 Fragen RJ, Avram MJ. Barbiturates. In: Miller RD, ed. Anesthesia. New York: Churchill Livingstone, 1994:22946.

19 Cordero L, Hon EH. Neonatal bradycardia following nasopharyngeal stimulation. F Pediatr 1971;78:441.

20 King BD, Harris LC, Greifenstein FE, et al. Reflex circulatory responses to direct laryngoscopy and tracheal intubation performed during general anesthesia. Anesthesiology 1951;12:556.

21 Tomori Z, Widdecombe JG. Muscular, bronchomotor and cardiovascular reflexes elicited by mechanical stimulation of the respiratory tract. F Physiol 1969;200:25.

22 Kiersey DK, Bickford RG, Faulkner A. Electroencephalographic patterns produced by thiopental sodium during surgical operations : description and classification. $\mathrm{Br} F$ Anaesth 1951;23:141.

23 Eckstein JW, Hamilton WK, McCammond JM. The effect of thiopental on peripheral venous tone. Anesthesiology 1961;22:525. 